\title{
Exercise and health in frail elderly people: a review of randomized controlled trials
}

\author{
Philipe de Souto Barreto
}

Received: 24 May 2009 / Accepted: 20 August 2009/Published online: 1 September 2009

(C) European Group for Research into Elderly and Physical Activity (EGREPA) 2009

\begin{abstract}
Frailty is a physiological syndrome that increases the risk of poor health. Although some research has been conducted to study the benefits of physical exercise in frail elderly populations, different operational definitions of frailty have been used, and this makes the studies difficult to compare. The present review was aimed at examining the influence of exercise on health in frail older adults. Studies using randomized controlled trials that administered an exercise program to a frail elderly population and that had an operational definition of frailty were selected from publications between January 2000 and October 2008. Information about the study population, frailty criteria, exercise program, principles of exercise training, randomization procedures, main and secondary outcome measures, study follow-up, and control group characteristics was taken from these studies, and the results from a final sample of 28 articles are discussed. Exercise training seems to be a safe and effective tool for promoting and maintaining optimal health levels in a wide variety of vulnerable older adults. However, the lack of studies on a well-defined frail older adult sample with selection procedures based on current knowledge in this field does not allow us, at the present time, to conclude that exercise influences health in this population. Further research is needed to confirm the benefits of exercise on health in frail older adults. The study population must be selected based on current knowledge in the area of frailty, and the design of the exercise program must be based on principles of training.
\end{abstract}

P. de Souto Barreto $(\triangle)$

CNRS UMR 6578 Laboratoire d'Anthropologie BioCulturelle, Faculté de Médecine-Secteur Nord, Université de la Méditerranée, CS80011, Bd Pierre Dramard,

13344 Marseille Cedex 15, France

e-mail: philipebarreto81@yahoo.com.br
Keywords Frailty Exercise - Older adult . Randomized controlled trial $\cdot$ Health

\section{Introduction}

Frailty is a syndrome (sometimes with subclinical signs) that disturbs the function of several physiologic systems $[1,2]$. This phenomenon has diverse etiological factors, possibly arising due to the interaction of biological (including genetics [3]), cognitive, social, clinical, psychological, and environmental aspects [4]. Researchers have been focusing their efforts in the study of the physical components of this syndrome, the so-called physical frailty. This review focuses on physical frailty. This health condition is directly associated with age [5] and enhances the risk of falls, hospitalization, morbidity, disability, and mortality $[6,7]$. Its prevalence varies among researches, but in general, it is between $7 \%$ and $32 \%$ [8]. Despite its importance for a successful aging, there is a lack of consensus regarding operational definitions of frailty and "frail" older adults, which diminishes comparability among studies. Although there is a historical confusion in scientific publications concerning the usage of frailty, comorbidity, and disability [9], with the two latter often being utilized as a synonym for the former, current knowledge indicates that frailty is a singular entity different from disability and comorbidity [10, 11]. Frail older adults often present a comorbidity condition and functional limitations [6], which indicate a strong linkage among these conditions. Although the debate concerning operational definitions of frailty continues, the most utilized definition is the one proposed by Fried et al. [6]. These authors defined frailty as meeting at least three of the following five indicators: (1) low physical activity 
level; (2) weakness; (3) slowness; (4) shrinking; and (5) poor endurance and energy.

A physically inactive lifestyle decreases cardiorespiratory endurance, flexibility, strength, and mobility ability (balance and muscular coordination). In this sense, physical inactivity (or a low level of physical activity) is one of the most frequent indicators of frailty found in studies [12]. Growing scientific evidence has shown the influence of exercise on frailty [13]. However, the different exercise-related regimens (type, frequency, intensity, and duration) and some inconsistencies found in randomized controlled trials (RCT), such as differences in operational definitions of frailty or no definition at all of a supposed frail elderly population, make comparisons among researches difficult and add to further complications in the debate on this subject.

The aim of this review was to study the influence of exercise on health in elderly people ( $\geq 60$ years old) defined as "frail." In this way, RCTs that administered an exercise program to a frail older adult population and that presented an operational definition of frailty were compiled, and results were discussed. Due to relatively recent advances in research on the frailty phenomenon, the present article selected RCTs published from January 2000 onwards.

\section{Methods}

\section{Search strategy}

Electronic searches were made in October 2008, utilizing three electronic databases: PubMed, Cochrane Library, and SciELO. Words for searches were entered in blocks of four, each block with a specific terminology (related to: exercise, elderly, frailty, and RCT). Similar to the recent systematic review on frailty made by Chin A Paw et al. [13], exerciserelated terms (e.g., exercise, physical activity, and training) were exhaustively interchanged in AND combinations with age-related (e.g., older adult, elderly, oldest, very old, and elders), frailty-related (e.g., frailty, frail), and study designrelated words (e.g., randomized, randomization, controlled trial, intervention, evaluation study, and treatment outcome). Some articles from the author's database and articles cited for another review [13] on exercise and frailty ${ }^{1}$ were incorporated when they met inclusion criteria.

\footnotetext{
${ }^{1}$ The difference between the present review and the review written by Chin A Paw et al. [13] is that the former studied the impact of exercise programs on health (physiological, physical function, and psychological outcomes) in elderly population with a definition of frailty, whereas the latter studied physical performance as measured by performance-based tests of physical function in elderly people labeled as "frail" (a definition of frailty was not mandatory for inclusion in the study).
}

Inclusion criteria

The inclusion criteria were: (1) RCT study design; (2) presence of at least one exercise group and one control group (receiving no intervention, non-exercise activities, or light intensity exercise such as a flexibility exercise program); (3) exercise intervention was not overlapped for another intervention; and (4) to present an operational definition of frailty. All full-text articles written in English, French, Spanish, and Portuguese were searched. However, all articles found for this review had been written in English, except for one article written in Spanish that was excluded because it was not a RCT.

\section{Procedures}

After electronic searches, articles with frailty-related words in the title were immediately accessed. Abstract and, if necessary, the methods topic of the text were read. This methodology allowed the author to immediately eliminate articles out of scope or that did not meet inclusion criteria. If inclusion criteria were met, with a frailty definition clearly indicated in the text, articles were selected $(n=13$ [14-26]). If complementary information were needed, the authors were contacted by e-mail $(n=15$; electronic address for one author was unavailable). Authors that did not answer the first e-mail were contacted once more 10 days after the first e-mail. Articles that met inclusion criteria were selected $(n=8$ [27-34]). Studies in which full text was not available but that were possible candidates to meet inclusion criteria were preselected, and their authors were contacted by e-mail to provide full text. From the nine authors contacted, seven sent their articles, but just two articles $[35,36]$ met inclusion criteria and were selected. Two articles $[37,38]$ cited in another review about exercise effectiveness in frail older adults [13] were selected for the present study. Finally, three studies [39-41] from the author's personal database took part in this review. Articles selected from other reviews and from the author's personal database did not necessarily have the terms "frail" or "frailty" in the title, but authors of these articles defined the study sample as frail in the text.

Information about study population, frailty criteria, exercise program, principles of exercise training, randomization, main and secondary outcome measures, study follow-up, and control group characteristics were extracted from each article. Some exercise regimen-related principles of training (progression, individualization, frequency, duration, intensity, and specificity - the latter was achieved when the exercise program was theoretically able to induce improvements on main outcome measures. For example, if strength is the main outcome measure of a study, and the exercise program consisted in resistance training in this 
case, exercise specificity was achieved) were evaluated herein on their "presence" or "absence" in the exercise program design (just when main outcome measures were physiological measures or physical performance-based measures). This evaluation, at least for frequency, duration, and intensity principles, was based on recommendations from the American College of Sports Medicine (ACSM) and the American Heart Association (AHA) for older adults [42] (for strength training, the number of repetitions per set and the number of exercises per session were not evaluated). Continuity was not evaluated because exercise adherence is a very complex health behavior, and it is not the aim of this study. Balance/coordination and taskspecific exercises do not have any precise prescription regarding frequency, duration, and intensity in elderly population [42]; that is why, they were considered as "present" herein if those exercises were met, at least twice a week during $20 \mathrm{~min}$ in a moderate to high intensity (as described by authors).

\section{Results}

The final sample was composed of 28 articles. Table 1 shows all studies arranged in alphabetical order, with information concerning the study sample, randomization, dropouts, and the operational criteria of frailty. Six studies $[14,19,24,37,39,41]$ based their criteria of frailty, at least in part, on functional limitation degree as measured by activities of daily living (ADL) and instrumental activities of daily living; disability in executing ADLs was the principal criteria for two of them [39, 41]. Performance in some functional fitness tests was the most utilized criteria to define frailty, being present in at least 19 articles [14, 15, 19-28, 31-34, 36, 37, 40], while physiological measures were found to characterize frailty in nine studies $[14,19$, $20,22,25,26,34-36]$ and fall-related aspects in six studies [24, 28, 31-33, 38]. Nutritional status and metabolic aspects contributed to define a frail elderly population in six articles [16-18, 20, 29, 38], and a low physical activity level participated in ten definitions [16-18, 20, 22, 25, 26, 29, 34, 36].

Five articles [16-18, 20,29] presented an operational definition of frailty that is different from disability and in accordance with current knowledge about this syndrome. They met two or more of the following aspects: physiological (for example, handgrip strength as measured by a dynamometer) and metabolic (for example, unintentional weight lost) aspects, poor endurance and energy, physical performance or mobility factors (for example, walking speed), and physical inactivity.

Sample size varied from 13 [23] to $311[25,26,36]$ individuals among studies. In eight articles [16, 20, 22, 24-
$26,34,36]$, it was $\geq 200$ persons, and in five others [19, 23, $27,30,35]$, it was $\leq 46$. Mean age varied from 78.5 [17] to 86.8 [27] years of age.

Exercise program, study follow-up and intervention length, control group characteristics, and training principles can be seen in Table 2, arranged by type of exercise program. Most of the studies $(n=25)$ utilized supervised exercise training, and the other three articles chose home-based exercise [21, 24, 40]. Multicomponent training was the choice for nine studies [14, 15, 19, 28, 29, 31-33, 37]. Tai chi exercise [22, $25,26,34,36]$, resistance training $[23,24,27,35,38]$, as well as skills and functional training [16-18, 39, 41] were found in five articles, and balance/gait/coordination in four other studies [20, 21, 30, 40]. Intervention length varied from a 48 -week period with tai chi exercise $[22,25,26,36]$ to a 10 -week intervention with resistance/balance program [24, 27, 35] or multicomponent training [31-33]. Control group characteristics also varied among studies, but most of them utilized group activities (either low-intensity exercise - commonly focused on flexibility or social groups) to prevent influence from "socializing and attention effects" on the results achieved. Regarding training principles, exercise specificity and frequency were the most prevalent principles being found in all studies followed by exercise individualization (except for one study [27]); exercise intensity was the least prevalent principle being "present" in 12 articles [14, 16, 19, 24, 28, 29, 31, 32, 35, 38, 39, 41].

Table 3 presents the main outcome measures and the secondary outcome measures, as well as respective results, arranged by type of main outcome measures. The results indicate significant differences that favored participants in the exercise group with relation to subjects in the control group. For some studies, results of secondary outcome measures were not reported because either there were no secondary outcome measures or authors did not clearly specify those measures in the articles. Most of the articles had physical function (self-reported and performancebased) and mobility ability measures as the main outcome of the study [14-16, 21, 27-32, 34, 36-41] followed by physiological measures [14, 16, 18, 19, 23, 29, 32, 34, 35, $38,41]$; psychological and mental aspects were found in seven articles [17, 22, 24, 25, 33, 34, 38] and fall risk in three studies [20, 24, 26]. Four articles [17, 24, 31, 38] did not present any difference between exercisers and control group participants regarding the results of the main outcome measures. Just in two studies, exercise training had a negative effect for frail elderly regarding main outcome measure (fall-risk [20] and cardiovascular endurance [34]). Results on secondary outcome measures were not achieved for six $[17,18,21,24,28,34]$ of the 14 studies concerned (i.e., those that clearly presented secondary outcome measures). 


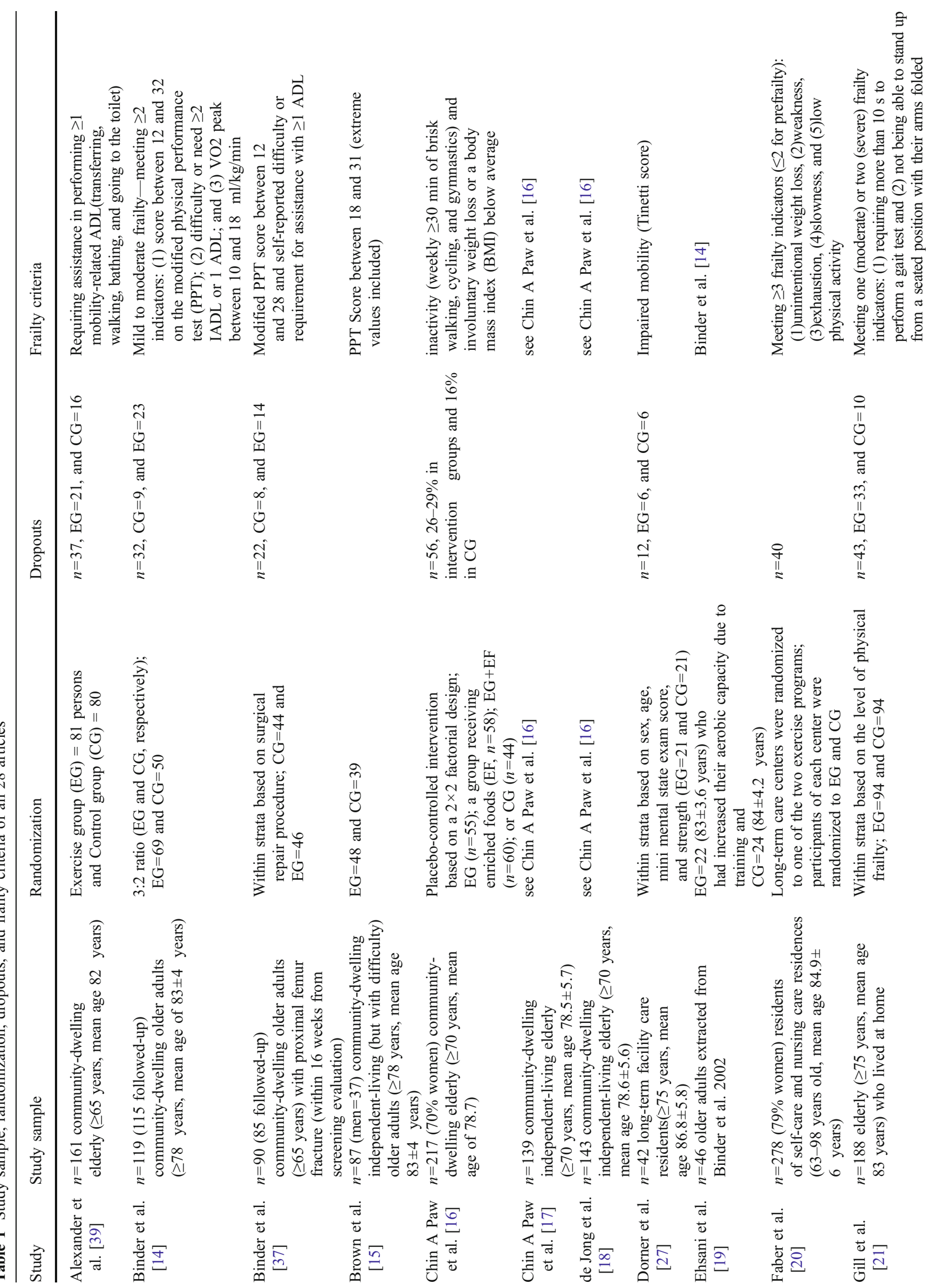




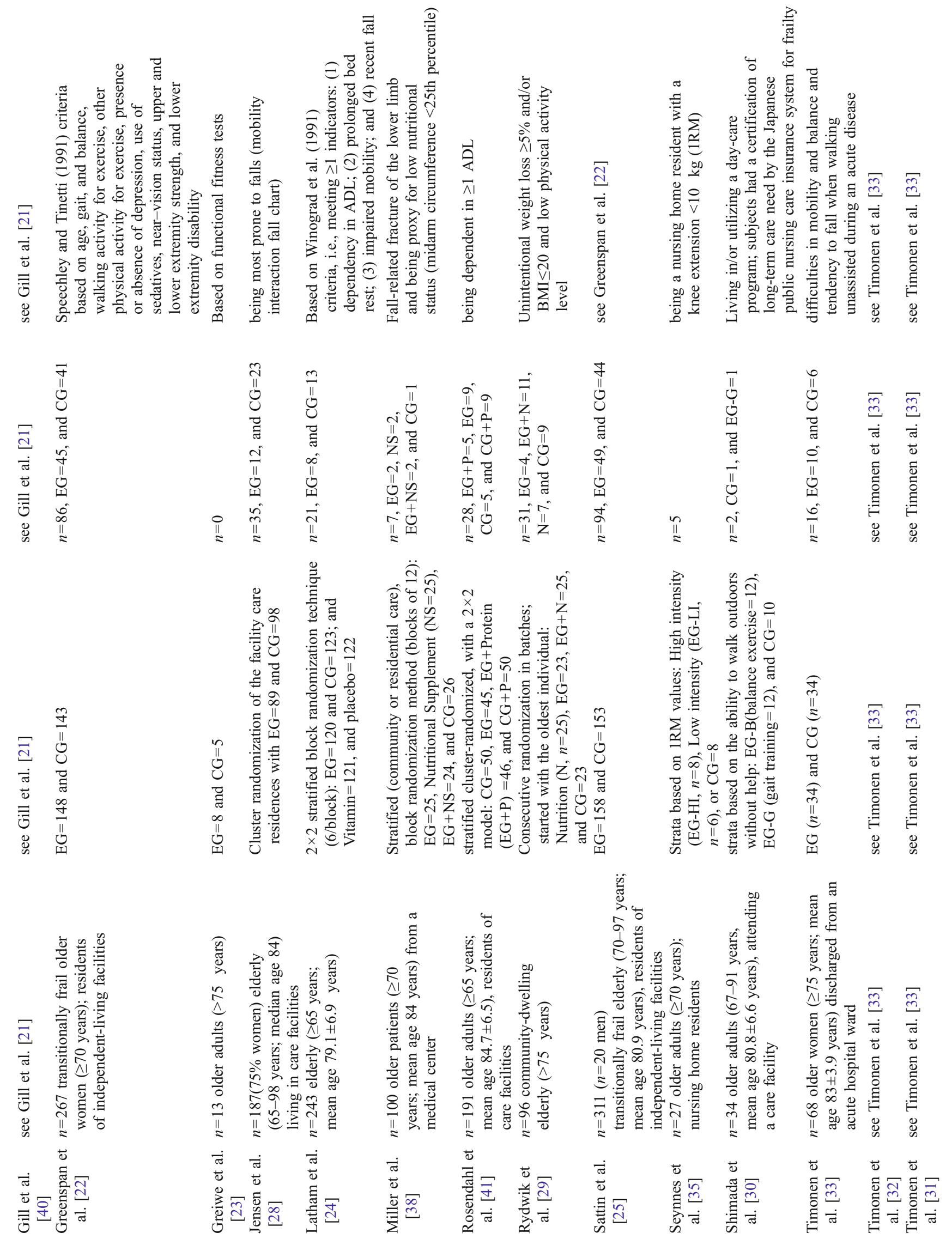




\section{Discussion}

This review provides some evidence of exercise effectiveness on health in a population of older adults defined as frail. The results presented for the analyzed 28 articles show that physiological factors, as well as functional fitness (FF, especially performance-based measures) and mobility ability (balance and coordination) and psychological aspects may be improved by exercise intervention in several vulnerable elderly populations.

Frailty criteria varied very much among studies. It is not surprising that the operational definition of frailty was not the same among articles because frailty is a recent subject in this research area. Furthermore, a standardized criterion of frailty has not yet been achieved by experts in this field [43]. Although it is very early to propose a single definition of frailty and then to standardize its operational criteria, efforts must be done to clearly define a frail elderly sample based on current knowledge in this field, thus, dissociating this syndrome from "disability." Just five articles [16-18, $20,29]$ presented an operational definition of frailty in accordance with current knowledge about this syndrome. In these studies, exercise was able to improve FF, mobility, lean body mass, and strength. One of them [20] presented contradictory results (FF and risk of falls were improved in prefrail older adults, but the opposite results were found among frail individuals). Although these results suggest a positive influence of exercise on health in frail older adults, it is too early to support this. More RCT studies are needed to confirm exercise benefits in a well-defined frail elderly population.

Future RCT researches on physical frailty must pay attention to the selection process of the study sample. At least three studies [27, 28, 41] had a sample partially composed of cognitively impaired or demented individuals. As indicated by Ferrucci et al. [44], frailty as a result of reduced cognition is considered a distinct clinical entity, although, decline in cognition may be found in frail persons. Intervention length is also a very important aspect for achieving improvements by exercise intervention. Frail older adults constitute a more vulnerable population when compared to healthy elderly; then, to provide improvements in physiological factors (e.g., strength, cardiovascular endurance, and flexibility), as well as in FF, they may need much time to adapt to and to progress in exercise volume.

Multicomponent training was the most utilized type of exercise program followed by tai chi, and skills and functional training, and resistance exercises. However, some incoherencies in relation to exercise programs were found. First, some studies did not clearly expose the exercise regimen-related principles of training. Exercise frequency, intensity (and how it is monitored), specificity, progression, individualization, and session duration are 
Table 2 Exercise program, study follow-up, control group characteristics, and training principles

\begin{tabular}{|c|c|c|c|c|}
\hline Study & Exercise program & $\begin{array}{l}\text { Study follow-up } \\
\text { and intervention } \\
\text { length }\end{array}$ & Control group characteristics & Training principles \\
\hline $\begin{array}{l}\text { Binder et } \\
\text { al. [14] }\end{array}$ & $\begin{array}{l}\text { Supervised multicomponent training } \\
\text { (flexibility, balance, coordination, } \\
\text { speed of reaction, strength, and } \\
\text { endurance), three times per week } \\
\text { during } 9 \text { months }\end{array}$ & 9 months & $\begin{array}{l}\text { Low-intensity home-based } \\
\text { training (flexibility); } \\
1 \mathrm{~h} / \text { session, two to three times } \\
\text { per week for } 9 \text { months }\end{array}$ & $\begin{array}{l}\text { Frequency, duration, intensity, } \\
\text { progression, individualization, } \\
\text { and specificity }\end{array}$ \\
\hline $\begin{array}{l}\text { Ehsani et } \\
\text { al. [19] }\end{array}$ & see Binder et al. [14] & $\begin{array}{l}\text { see Binder et al. } \\
{[14]}\end{array}$ & see Binder et al. [14] & $\begin{array}{l}\text { progression, individualization, } \\
\text { frequency, duration, intensity, } \\
\text { and specificity }\end{array}$ \\
\hline $\begin{array}{l}\text { Brown et } \\
\text { al. [15] }\end{array}$ & $\begin{array}{l}\text { Supervised low-intensity multicomponent } \\
\text { training, three times per week for } \\
3 \text { months (flexibility, balance, body } \\
\text { handling skills, speed of reaction, } \\
\text { coordination, and strength) }\end{array}$ & 3 months & $\begin{array}{l}\text { Low-intesity home-based } \\
\text { exercise (flexibility); } \\
1 \text { h/session, three times per } \\
\text { week for } 3 \text { months }\end{array}$ & $\begin{array}{l}\text { Progression, frequency, } \\
\text { individualization, and } \\
\text { specificity }\end{array}$ \\
\hline $\begin{array}{l}\text { Jensen et } \\
\text { al. [28] }\end{array}$ & $\begin{array}{l}\text { 11-week supervised multicomponent } \\
\text { exercise program (balance, ambulation, strength, } \\
\text { endurance, flexibility, and } \\
\text { safe movement behavior) executed, in general, two } \\
\text { to three } \\
\text { times per week, } 1-3 \mathrm{~h} / \text { week }\end{array}$ & $\begin{array}{l}\text { 11-week } \\
\text { intervention, } \\
\text { 9-month } \\
\text { follow-up }\end{array}$ & Usual care & $\begin{array}{l}\text { Progression, individualization, } \\
\text { intensity, duration, frequency, } \\
\text { and specificity }\end{array}$ \\
\hline $\begin{array}{l}\text { Rydwik et } \\
\text { al. [29] }\end{array}$ & $\begin{array}{l}\text { Supervised multicomponent exercise } \\
\text { program (endurance, strength, and } \\
\text { balance), } 1 \mathrm{~h} / \text { session, } \\
\text { two times per week for } 12 \text { weeks }\end{array}$ & $\begin{array}{l}\text { 3-month } \\
\text { intervention, } \\
\text { 9-month } \\
\text { follow-up }\end{array}$ & $\begin{array}{l}\text { General advice on physical } \\
\text { training and diet }\end{array}$ & $\begin{array}{l}\text { Progression, individualization, } \\
\text { intensity, duration, frequecy, } \\
\text { and specificity }\end{array}$ \\
\hline $\begin{array}{l}\text { Timonen } \\
\text { et al. [33] }\end{array}$ & $\begin{array}{l}\text { Supervised multicomponent exercise } \\
\text { program (resistance training and } \\
\text { functional exercises), two times per } \\
\text { week, } 90 \mathrm{~min} / \mathrm{session} \text {, for } 10 \text { weeks }\end{array}$ & $\begin{array}{l}\text { 10-month } \\
\text { intervention, } \\
\text { 9-month } \\
\text { follow-up after } \\
\text { intervention }\end{array}$ & $\begin{array}{l}\text { Requested to perform a home- } \\
\text { based functional exercise } \\
\text { program, two to three times per } \\
\text { week }\end{array}$ & \\
\hline $\begin{array}{l}\text { Timonen } \\
\text { et al. } \\
\text { [32] }\end{array}$ & see Timonen et al. [33] & $\begin{array}{l}\text { see Timonen } \\
\text { et al. [33] }\end{array}$ & see Timonen et al. [33] & $\begin{array}{l}\text { Progression, individualization, } \\
\text { intensity, duration, frequency, } \\
\text { and specificity }\end{array}$ \\
\hline $\begin{array}{l}\text { Timonen } \\
\text { et al. } \\
\text { [31] }\end{array}$ & see Timonen et al. [33] & $\begin{array}{l}\text { see Timonen } \\
\text { et al. [33] }\end{array}$ & see Timonen et al. [33] & $\begin{array}{l}\text { Progression, individualization, } \\
\text { intensity, duration, frequency, } \\
\text { and specificity }\end{array}$ \\
\hline $\begin{array}{l}\text { Binder et } \\
\text { al. [37] }\end{array}$ & $\begin{array}{l}6 \text { months of supervised multicomponent } \\
\text { training (flexibility, balance, } \\
\text { coordination, movement speed, } \\
\text { strength, and endurance), 45-90 } \\
\text { min/session (with possible breaks), } \\
\text { three times per week }\end{array}$ & 6 months & $\begin{array}{l}\text { Low-intesity home-based exercise } \\
\text { (flexibility); } 1 \mathrm{~h} / \text { session three times } \\
\text { per week for } 6 \text { months }\end{array}$ & $\begin{array}{l}\text { Progression, individualization } \\
\text { frequency, specificity, and } \\
\text { duration }\end{array}$ \\
\hline $\begin{array}{l}\text { Greenspan } \\
\text { et al. } \\
\text { [22] }\end{array}$ & $\begin{array}{l}\text { Supervised tai chi exercise (trunk rotation, } \\
\text { weight shifting, coordination, and gradual } \\
\text { narrowing of the lower extremity stance), } \\
\text { two times per week from } 10 \text { to } 50 \text { min for } \\
48 \text { weeks }\end{array}$ & 48 weeks & $\begin{array}{l}\text { wellness educational program } \\
\text { (instructions on falls-prevention, } \\
\text { exercise, balance diet, and } \\
\text { nutrition) } 1 \mathrm{~h} / \text { week }\end{array}$ & \\
\hline $\begin{array}{l}\text { Sattin et } \\
\text { al. [25] }\end{array}$ & see Greenspan et al. [22] & $\begin{array}{l}\text { see Greenspan } \\
\text { et al. [22] }\end{array}$ & see Greenspan et al. [22] & \\
\hline $\begin{array}{l}\text { Wolf et al. } \\
\text { [26] }\end{array}$ & see Greenspan et al. [22] & $\begin{array}{l}\text { see Greenspan } \\
\text { et al. [22] }\end{array}$ & see Greenspan et al. [22] & $\begin{array}{l}\text { Progression, individualization, } \\
\text { duration, frequency, and } \\
\text { specificity }\end{array}$ \\
\hline $\begin{array}{l}\text { Wolf et al. } \\
\text { [36] }\end{array}$ & see Greenspan et al. [22] & $\begin{array}{l}\text { see Greenspan } \\
\text { et al. [22] }\end{array}$ & see Greenspan et al. [22] & $\begin{array}{l}\text { Progression, individualization, } \\
\text { frequency, duration, and } \\
\text { specificity (for some measures) }\end{array}$ \\
\hline $\begin{array}{l}\text { Wolf et al. } \\
\text { [34] }\end{array}$ & $\begin{array}{l}15 \text { weeks of supervised tai chi (two } \\
\text { times per week; subjects were } \\
\text { requested to try two times per day } \\
\text { for } 15 \text { min - not monitored) or balance } \\
\text { training (once per week) }\end{array}$ & $\begin{array}{l}\text { 15-month } \\
\text { intervention; } \\
\text { 4-month after } \\
\text { follow-up }\end{array}$ & $\begin{array}{l}\text { Discussions with a nurse (e.g., } \\
\text { sleep disorders), once a week, } \\
1 \mathrm{~h} / \text { session for } 15 \text { weeks }\end{array}$ & $\begin{array}{l}\text { Progression, frequency } \\
\text { individualization, and specificity }\end{array}$ \\
\hline $\begin{array}{l}\text { Greiwe et } \\
\text { al. }[23]\end{array}$ & $\begin{array}{l}\text { Supervised resistance training } \\
\text { program, three times per week for } \\
3 \text { months, } 50-90 \mathrm{~min} / \mathrm{session}\end{array}$ & 3 months & $\begin{array}{l}\text { Light stretching program } \\
\text { for } 3 \text { months }\end{array}$ & \\
\hline $\begin{array}{l}\text { Latham et } \\
\text { al. [24] }\end{array}$ & $\begin{array}{l}\text { Home-based resistance quadriceps } \\
\text { exercise (ankle cuff weights), three } \\
\text { times per week during } 10 \text { weeks }\end{array}$ & $\begin{array}{l}\text { 10-month } \\
\text { intervention, } \\
\text { 6-month } \\
\text { follow-up }\end{array}$ & $\begin{array}{l}\text { Frequency-matched telephone } \\
\text { calls and home visits }\end{array}$ & $\begin{array}{l}\text { Individualization, specificity, } \\
\text { intensity, and frequency }\end{array}$ \\
\hline $\begin{array}{l}\text { Dorner et } \\
\text { al. [27] }\end{array}$ & $\begin{array}{l}\text { three times per week, } 50 \mathrm{~min} / \mathrm{session} \text {, } \\
\text { of a supervised resistance exercise }\end{array}$ & 10 weeks & & $\begin{array}{l}\text { Frequency, duration, and } \\
\text { specificity }\end{array}$ \\
\hline
\end{tabular}


Table 2 (continued)

\begin{tabular}{|c|c|c|c|c|}
\hline Study & Exercise program & $\begin{array}{l}\text { Study follow-up } \\
\text { and intervention } \\
\text { length }\end{array}$ & Control group characteristics & Training principles \\
\hline $\begin{array}{l}\text { Seynnes et } \\
\text { al. [35] }\end{array}$ & $\begin{array}{l}\text { Supervised } 10 \text {-week resistance training } \\
\text { (knee muscles), three times per week }\end{array}$ & 10 weeks & $\begin{array}{l}\text { Placebo exercise (empty cuff } \\
\text { weights) }\end{array}$ & $\begin{array}{l}\text { Progression, individualization, } \\
\text { intensity, frequency, and } \\
\text { specificity }\end{array}$ \\
\hline $\begin{array}{l}\text { Miller et } \\
\text { al. [38] }\end{array}$ & $\begin{array}{l}\text { Supervised resistance training (hip } \\
\text { extensors and abductors, knee extensors, ankle } \\
\text { dorsi, and plantar-flexors), three } \\
\text { times per week, } 20-30 \mathrm{~min} / \mathrm{session} \text { for } \\
12 \text { weeks }\end{array}$ & 12 weeks & $\begin{array}{l}\text { Usual care. matched visits } \\
\text { ("attention effect"), three times } \\
\text { per week (weeks 1-6); and once } \\
\text { a week (weeks 7-12) }\end{array}$ & $\begin{array}{l}\text { Progression, individualization, } \\
\text { intensity, duration, frequency, } \\
\text { and specificity }\end{array}$ \\
\hline $\begin{array}{l}\text { Chin A } \\
\text { Paw et } \\
\text { al. }[16]\end{array}$ & $\begin{array}{l}\text { Supervised skills training program, } \\
\text { two times per week for } 17 \text { weeks, } \\
45 \mathrm{~min} / \text { session (focused on strength, } \\
\text { speed, endurance, flexibility, and } \\
\text { coordination) }\end{array}$ & 17 weeks & $\begin{array}{l}\text { social program, once or twice a week, } \\
90 \mathrm{~min} / \mathrm{session} \text { (adjustment for } \\
\text { socializing and attention effects) }\end{array}$ & $\begin{array}{l}\text { Progression, individualization, } \\
\text { duration, frequency, intesity, } \\
\text { and specificity }\end{array}$ \\
\hline $\begin{array}{l}\text { Chin A } \\
\text { Paw et } \\
\text { al. }[17]\end{array}$ & see Chin A Paw et al. [16] & $\begin{array}{l}\text { see Chin A Paw } \\
\text { et al. [16] }\end{array}$ & see Chin A Paw et al. [16] & \\
\hline $\begin{array}{l}\text { de Jong et } \\
\text { al. [18] }\end{array}$ & see Chin A Paw et al. [16] & $\begin{array}{l}\text { see Chin A Paw } \\
\text { et al. [16] }\end{array}$ & see Chin A Paw et al. [16] & \\
\hline $\begin{array}{l}\text { Alexander } \\
\text { et al. [39] }\end{array}$ & $\begin{array}{l}\text { Supervised bed- and chair-rise } \\
\text { task-specific training with emphasis on strength and } \\
\text { range of motion (proximal } \\
\text { upper and lower extremity, musculature, } \\
\text { and trunk), performed for } 12 \text { weeks, } \\
\text { three times per week, } 1 \mathrm{~h} / \text { session }\end{array}$ & 12 weeks & $\begin{array}{l}\text { 12-week exercise program focused on } \\
\text { flexibility, three times per week, } \\
1 \mathrm{~h} / \text { session }\end{array}$ & $\begin{array}{l}\text { Progression, individualization, } \\
\text { intensity, frequency, duration, } \\
\text { and specificity }\end{array}$ \\
\hline $\begin{array}{l}\text { Rosendahl } \\
\text { et al. [41] }\end{array}$ & $\begin{array}{l}\text { Supervised functional exercise program } \\
\text { (everyday tasks challenging leg strength, postural } \\
\text { stability, and gait ability) with } 45 \mathrm{~min} / \mathrm{session} \text {, five } \\
\text { times every } 2 \text { weeks for } 3 \text { months }\end{array}$ & $\begin{array}{l}\text { 3-month } \\
\text { intervention } \\
\text { and 6-month } \\
\text { follow-up }\end{array}$ & $\begin{array}{l}\text { Social activities (watching films, } \\
\text { reading, singing, and conversation) }\end{array}$ & $\begin{array}{l}\text { Progression, individualization, } \\
\text { frequency, duration, intensity, } \\
\text { and specificity }\end{array}$ \\
\hline $\begin{array}{l}\text { Faber et } \\
\text { al. [20] }\end{array}$ & $\begin{array}{l}\text { Supervised fall-preventive exercise } \\
\text { programs (focused on balance and } \\
\text { functional strength, and/or tai chi } \\
\text { principles), } 60 \mathrm{~min} / \mathrm{session} \text {, once a } \\
\text { week for } 4 \text { weeks, } \\
\text { and two times per week for } 16 \text { weeks. }\end{array}$ & $\begin{array}{l}\text { 20-week } \\
\text { intervention } \\
\text { and 52-week } \\
\text { follow-up }\end{array}$ & & $\begin{array}{l}\text { Progression, individualization, } \\
\text { duration, frequency, and } \\
\text { specificity }\end{array}$ \\
\hline $\begin{array}{l}\text { Gill et al. } \\
\text { [21] }\end{array}$ & $\begin{array}{l}\text { 6-month home-based exercise program } \\
\text { (balance once a day and leg and } \\
\text { arm-conditioning and strengthening three times per } \\
\text { week); participants received in average } 14.9 \text { visits of } \\
\text { a physical therapist }\end{array}$ & $\begin{array}{l}\text { 6-month } \\
\text { intervention } \\
\text { and } 12 \text {-month } \\
\text { follow-up }\end{array}$ & $\begin{array}{l}\text { educational program (attention } \\
\text { and health education), } 45-60 \\
\text { min/session for } 6 \text { months, with } \\
\text { visits of a health educator }\end{array}$ & $\begin{array}{l}\text { Individualization, specificity, and } \\
\text { frequency }\end{array}$ \\
\hline $\begin{array}{l}\text { Gill et al. } \\
\text { [40] }\end{array}$ & see Gill et al. [21] & $\begin{array}{l}\text { see Gill et al. } \\
\text { [21] }\end{array}$ & see Gill et al. [21] & $\begin{array}{l}\text { Individualization, specificity, and } \\
\text { frequency }\end{array}$ \\
\hline $\begin{array}{l}\text { Shimada } \\
\text { et al. [30] }\end{array}$ & $\begin{array}{l}\text { Two supervised exercise training } \\
\text { (balance and gait training), two to } \\
\text { three times per week during } \\
40 \mathrm{~min} / \mathrm{session} \text {, for } 12 \text { weeks }\end{array}$ & 12 weeks & Usual care & $\begin{array}{l}\text { Individualization, duration, } \\
\text { frequency, } \\
\text { and specificity }\end{array}$ \\
\hline
\end{tabular}

basic data indispensable to analyze exercise effectiveness. Thus, they have to be clearly exposed in the text even when the training program has been detailed elsewhere. This does not mean that some of the researches evaluated herein did not base their exercise program on principles of training, but these data were not precise enough to be extracted from the text.

Second, some inadequacies were found in exercise program design, which led some authors to suggest that exercise was not effective. In a home-based quadriceps resistance exercise program, Latham et al. [24] concluded that this "form of resistance exercise was harmful to patients, as evidenced by the higher incidence of musculoskeletal injuries." However, the exercise program started with a high-intensity resistance training (60-80\% of $1 \mathrm{RM})$, which probably led to musculoskeletal injuries. This kind of problem can be prevented if exercise training is taken in a gradual or stepwise approach [42] (principle of load progression) in the beginning of the intervention, which Latham et al. [24] did after participants' complaints. Moreover, this inadequacy may have negatively influenced results achieved in relation to self-rated physical health, a main outcome measure of the study (there was no difference between exercisers and control individuals). In the same way, Faber et al. [20] indicated that the "absence of significant positive training effects (regarding frail individuals) might also be attributed to inadequacy of training intensity, frequency, duration, and/or specificity of the exercise mode." Brown et al. [15] did not find any difference on flexibility between exercisers and control 
Table 3 Main outcome measures and secondary outcome measures with respective results

\begin{tabular}{ccc}
\hline Study & Main outcome measures & Results (main outcome) \\
\hline $\begin{array}{c}\text { Gill et al. } \\
\text { [21] }\end{array}$ & $\begin{array}{c}\text { Summary disability score } \\
\text { (performance in ADLs) }\end{array}$ & $\begin{array}{c}\text { Compared with CG subjects, EG } \\
\text { ones improved: disability scores } \\
\text { (moderately frail subgroup) }\end{array}$ \\
$\begin{array}{ccc}\text { Timonen } \\
\text { et al. }\end{array}$ & $\begin{array}{c}\text { ADL and IADL levels (Joensuu } \\
\text { classification) }\end{array}$ & differences were achieved \\
[31] & Modified PPT, functional status & Compared with CG subjects, \\
$\begin{array}{c}\text { Binder et } \\
\text { al. [37] }\end{array}$ & questionnaire (FSQ), and & EG ones improved: modified \\
& ADL instruments & PPT score, and FSQ score
\end{tabular}

Gill et al. Self-reported IADLs; mobility [40] (modified performance oriented mobility assessment—POMA); timed rapid gait and chair stands; and modified PPT

Dorner et Muscle function, cognitive

al. [27] function (MMSE)

Alexander Bed- and chair-rise task perforet al. mance ability and time taken [39] to rise

\section{Jensen et Ambulation (functional}

al. [28] ambulations category scale), usual, and maximum gait speed, balance (BBT), and step height.

Shimada Balance (one leg standing,

et al. [30] functional reach, manual perturbation test, functional balance scale, and POMA) and gait (timed up-and-go [TUG], and stair climbing/descending)

Wolf et al. FF (e.g., gait speed, functional [36] reach test, timed chair-stand, timed $360^{\circ}$-turn, and single limb stance)

Binder et Modified PPT, VO2 peak, and

al. [14] ADL measures (FSQ)

Brown et PPT, balance, gait, strength,

al. [15] flexibility, speed of reaction, and coordination, peripheral sensation

Chin A FF tests (e.g., balance and gait

Paw et speed), physical fitness (e.g.,

al. [16] strength, flexibility, and

reaction time), self-rated disabilities in ADLs
Compared with CG subjects, EG ones improved: IADL, timed rapid gait, mobility, timed chair stands, and modified PPT

Compared with CG subjects, EG ones improved: muscle function

Compared with CG subjects, EG ones improved: bed- and chair-rise task performance ability and time taken to rise

Compared with CG subjects, EG ones improved: step height and ambulation (not decreased), usual, and maximum gait speed Compared with CG subjects, EG ones improved: balance

Compared with CG subjects, EG ones improved: chairtraining)

Compared with CG subjects, EG ones improved: modified PPT score, $\mathrm{VO} 2$ peak, and FSQ score

Compared with CG subjects, EG ones improved: PPT score, stregth, balance, and gait

Compared with CG subjects, EG ones improved: FF score (mainly chair-stand, touching toes, and walking speed) and physical performance when adjusted for baseline scores stand (after 4 and 8-month
Strength knee extension (KET) and flexion torque (KFT); gait; balance (progressive Romerg Test, Berg balance Test (BBT), and single-limb stance); body composition; quality of life (SF-36), and a modified hip rating questionnaire (HRQ)

Lean body mass, ADLs, mobility (Tinetti score), and depression

strength, range of motion (ROM), and trunk lateral balance

Risk of falling

Height, weight, BMI, Systolic, diastolic blood pressure, and resting heart rate

KET and KFT; balance (leg stance time, and BBT); change health subscale of SF-36; and weight
Compared with CG subjects, EG ones improved: KET (both limbs), 1RM (knee extension and flexion, seated bench press, seated row, leg press, and biceps curl), fast walking speed, Berg balance score, single-limb stance time (fractured leg), HRQ score, and the change in health and physical function subscale scores of the SF-36

Compared with CG subjects, EG ones improved: LBM

Compared with CG subjects, EG ones improved: trunk lateral balance, ROM, and strength (mainly in trunk region)

No differences were achieved
Compared with CG subjects, EG ones improved: BMI, SBP, and resting heart rate

Compared with CG subjects, EG ones improved: KET, KFT, balance (one leg stance time and BBT), and change in health subscale SF-36 
Table 3 (continued)

\begin{tabular}{|c|c|c|c|c|}
\hline Study & Main outcome measures & Results (main outcome) & Secondary outcome measures & Results (secondary outcome) \\
\hline $\begin{array}{l}\text { Rydwik et } \\
\text { al. [29] }\end{array}$ & $\begin{array}{l}\text { Muscle strength, FF (30-second } \\
\text { chair-stand, balance tandem } \\
\text { and one leg stance, TUG, and } \\
\text { gait speed), ADLs (functional } \\
\text { independence measure), and } \\
\text { IADLs (instrumental activity } \\
\text { measures) }\end{array}$ & $\begin{array}{l}\text { Compared with CG subjects, } \\
\text { EG ones improved: strength; } \\
\text { no differences persisted } \\
\text { achieved } 9 \text { months after } \\
\text { randomization }\end{array}$ & & \\
\hline $\begin{array}{l}\text { Timonen } \\
\text { et al. [32] }\end{array}$ & $\begin{array}{l}\text { Knee (KET) and hip abduction } \\
\text { strength, balance (14-item BBT), } \\
\text { and maximal walking speed }\end{array}$ & $\begin{array}{l}\text { Compared with CG subjects, } \\
\text { EG ones improved: hip } \\
\text { abduction strength, KET, } \\
\text { balance, and walking speed }\end{array}$ & & \\
\hline $\begin{array}{l}\text { Wolf et al. } \\
\text { [34] }\end{array}$ & $\begin{array}{l}\text { Strength, flexibility, } \\
\text { cardiovascular endurance, body } \\
\text { composition, IADL score, } \\
\text { depression, and fear of falling }\end{array}$ & $\begin{array}{l}\text { Compared with CG subjects, EG } \\
\text { ones improved: left handgrip } \\
\text { strength and systolic blood } \\
\text { pressure (TC group); however, } \\
\text { TC exercisers reduced the } \\
\text { distance ambulated } \\
\text { (cardiovascular endurance) }\end{array}$ & Time-specific risk for falls & No differences were achieved \\
\hline $\begin{array}{l}\text { Miller et } \\
\text { al. [38] }\end{array}$ & $\begin{array}{l}\text { Weight, quadriceps strength, } \\
\text { usual gait speed, and quality } \\
\text { of life (SF-12) }\end{array}$ & No differences were achieved & & \\
\hline $\begin{array}{l}\text { Rosendahl } \\
\text { et al. } \\
\text { [41] }\end{array}$ & $\begin{array}{l}\text { Balance (BBT), gait, and lower } \\
\text { limb strength (1RM or chair-- } \\
\text { stand) }\end{array}$ & $\begin{array}{l}\text { Compared with CG subjects, } \\
\text { EG ones improved: usual gait } \\
\text { speed, balance, and lower- } \\
\text { limb strength }\end{array}$ & & \\
\hline $\begin{array}{l}\text { de Jong et } \\
\text { al. [18] }\end{array}$ & $\begin{array}{l}\text { Body composition (dual-energy } \\
\text { X-ray absorptiometry) }\end{array}$ & $\begin{array}{l}\text { Compared with CG subjects, } \\
\text { EG ones improved: lean body } \\
\text { mass; exercise had no effect } \\
\text { on bone parameters }\end{array}$ & $\begin{array}{l}\text { Weight, BMI, waist and } \\
\text { hip, and waist-to-hip } \\
\text { circumferences }\end{array}$ & No differences were achieved \\
\hline $\begin{array}{l}\text { Ehsani et } \\
\text { al. [19] }\end{array}$ & $\begin{array}{l}\text { Maximal cardiac output (heart } \\
\text { rate, left ventricular [LV] } \\
\text { function), arteriovenous } \mathrm{O} 2 \\
\text { content difference }\end{array}$ & $\begin{array}{l}\text { Compared with CG sujects, EG } \\
\text { ones improved: cardiac } \\
\text { output, LV stroke work (peak } \\
\text { effort) and peak heart rate }\end{array}$ & $\begin{array}{l}\text { Body composition LBM and } \\
\text { weight }\end{array}$ & $\begin{array}{l}\text { Compared with CG sujects, EG } \\
\text { Ones improved: LBM }\end{array}$ \\
\hline $\begin{array}{l}\text { Greiwe et } \\
\text { al. [23] }\end{array}$ & $\begin{array}{l}\text { Cytokine tumor necrosis factor } \alpha \\
(\mathrm{TNF}-\alpha) \text { level, protein synthesis } \\
\text { rate, and lipoprotein lipase } \\
\text { (LPL) }\end{array}$ & $\begin{array}{l}\text { EG had decreased skeletal } \\
\text { muscle TNF- } \alpha \text { and increased } \\
\text { LPL expression and protein } \\
\text { synthesis rate }\end{array}$ & & \\
\hline $\begin{array}{l}\text { Seynnes et } \\
\text { al. [35] }\end{array}$ & Knee extension strength (KET) & $\begin{array}{l}\text { Compared with CG subjects } \\
\text { and EG ones improved: KET }\end{array}$ & $\begin{array}{l}\text { Functional limitations (6-min } \\
\text { walking, chair-rising, and stair } \\
\text { climbing) and self-reported dis- } \\
\text { ability (French version of health } \\
\text { assessment questionnaire and } \\
\text { disability index subscale) }\end{array}$ & $\begin{array}{l}\text { Compared with CG subjects, } \\
\text { EG ones improved: 6-min } \\
\text { walking (just for EG-HI), } \\
\text { chair-rising, and stair } \\
\text { climbing }\end{array}$ \\
\hline $\begin{array}{l}\text { Chin A } \\
\text { Paw et } \\
\text { al. [17] }\end{array}$ & $\begin{array}{l}\text { Subjective well being } \\
\text { (subscales: health, self-respect, } \\
\text { morale, optimism, and } \\
\text { contacts) }\end{array}$ & No differences were achieved & Self-rated health & No differences were achieved \\
\hline $\begin{array}{r}\text { Greenspan } \\
\text { et al.[22] }\end{array}$ & $\begin{array}{l}\text { Perceived health status } \\
\text { (sickness impact profile) and } \\
\text { self-rated health }\end{array}$ & $\begin{array}{l}\text { Compared with CG subjects } \\
\text { and EG ones improved: } \\
\text { perceived health status } \\
\text { (physical dimension, mainly, } \\
\text { ambulatory category) }\end{array}$ & & \\
\hline $\begin{array}{l}\text { Sattin et } \\
\text { al. [25] }\end{array}$ & $\begin{array}{l}\text { Fear of falling (Activities- } \\
\text { Specific Balance Confidence } \\
\text { Scale }[\mathrm{ABC}] \text {, and fall efficacy } \\
\text { scale) }\end{array}$ & $\begin{array}{l}\text { Compared with CG subjects } \\
\text { and } E G \text { ones improved: fear } \\
\text { of falling }(\mathrm{ABC})\end{array}$ & & \\
\hline $\begin{array}{l}\text { Timonen } \\
\text { et al. }\end{array}$ & $\begin{array}{l}\text { mood (Zung self-rating } \\
\text { depression scale) }\end{array}$ & $\begin{array}{l}\text { Compared with CG subjects } \\
\text { and EG ones improved: mood }\end{array}$ & & \\
\hline
\end{tabular}

[33] 
Table 3 (continued)

\begin{tabular}{|c|c|c|c|c|}
\hline Study & Main outcome measures & Results (main outcome) & Secondary outcome measures & Results (secondary outcome) \\
\hline $\begin{array}{l}\text { Latham et } \\
\text { al. [24] }\end{array}$ & $\begin{array}{l}\text { Self-rated physical health } \\
\text { (physical component of the } \\
\text { SF-36) and risk of falls }\end{array}$ & No differences were achieved & $\begin{array}{l}\text { ADL, physical performance } \\
\text { (strength, balance, mobility, } \\
\text { and gait speed), FF, fear of } \\
\text { falling, social activities, and } \\
\text { mental health }\end{array}$ & No differences were achieved \\
\hline $\begin{array}{l}\text { Faber et } \\
\text { al. [20] }\end{array}$ & Fall risk & $\begin{array}{l}\text { Fall risk in prefrail subjects } \\
\text { (EGs) decreased but it } \\
\text { increased in frail elderly } \\
\text { (EGs) }\end{array}$ & $\begin{array}{l}\text { mobility (POMA) and FF and } \\
\text { self-reported disability (ADL, } \\
\text { and IADL) }\end{array}$ & $\begin{array}{l}\text { Compared with CG subjects } \\
\text { and EG ones improved: } \\
\text { mobility (POMA score) and } \\
\text { FF (prefrail subgroup); FF } \\
\text { decreased in frail subgroup } \\
\text { (EG) }\end{array}$ \\
\hline $\begin{array}{l}\text { Wolf et al. } \\
\text { [26] }\end{array}$ & Fall risk & $\begin{array}{l}\text { EG presented lower fall risk } \\
\text { from months } 4 \text { to } 12\end{array}$ & & \\
\hline
\end{tabular}

subjects, though the control group met an exercise program focused on range of motion, $1 \mathrm{~h}$ per session, three times a week (more than the minimum prescribed for older adults by ACSM and AHA) during the intervention period. Furthermore, both groups presented improvements when compared to baseline assessments, which suggests that the exercise program was able to improve flexibility. In the study of Wolf et al. [34], tai chi exercisers reduced their cardiovascular endurance (distance ambulated in a 12-min walking test) after a 15 -week period intervention. In a review study, Kuramoto et al. [45] indicated that "Tai Chi may be an additional form of aerobic exercise." However, two meta-analyses concerning the effects of tai chi on aerobic capacity $[46,47]$ concluded that the average effect size was small and nonsignificant for subjects enrolled in the experimental studies. Further studies are needed to know the real influence of tai chi on cardiovascular endurance.

Third, several of these studies were, apparently, part of the same trial (same sample and exercise training) with different main outcome measures. Thus, the exercise program may have been more adequate for some of the outcome measures in one study than it was for other measures in another study. This may have influenced some of the results. In those cases, the absence of positive results of exercise training on outcome measures does not necessarily mean an absence of the effect (see comments about Brown et al. [15] just above).

Furthermore, general guidelines for balance exercise, such as ACSM and AHA, are not precise enough to guide the design of RCT studies. As indicated by Nelson et al. [42], "the preferred types, frequency, and duration of balance training are unclear," which makes it difficult to design a study based on current recommendations to improve balance in a frail older adult population. It may partially explain the ineffectiveness of intervention in some trials (in main outcome measures [20], as well as in secondary ones [24]).

The present study has some limitations. First, in order to be selected through electronic searches, articles had to contain the terms "frail" or "frailty" in the title, which may have limited the final sample. Second, articles had to present an operational definition of frailty to meet the inclusion criteria, which may also have reduced the final sample. Finally, the several operational definitions of frailty found among studies characterized a large range of vulnerable populations. It is possible that other studies, with similar samples, have not been found by electronic searches and, therefore, were not included because authors did not label their population as "frail."

However, to the author's knowledge, it is the first review that compiles RCTs that studied exercise effects on health in frail elderly populations with a defined criterion of frailty. The study methodology, with direct contacts with authors of the original RCTs to clarify some points of the trials, was a strong aspect of the present review. This permitted the compilation of articles that had not presented a clear definition of frailty in the text.

\section{Conclusion}

Frailty is a physiological syndrome that increases the risk of poor health. Researches that studied exercise benefits in frail elderly populations utilized different operational definitions of frailty, which renders comparisons among them difficult to make. However, an increasing amount of evidence supports the affirmation that exercise training is an important tool to improve health in various at-risk populations. This review suggests that exercise is able to improve physiological factors (e.g., strength, cardiovascular endurance, and flexibility), as well as FF (especially 
performance-based measures) and mobility ability (balance and gait), and psychological (e.g., perceived health status, fear of falling, and mood) aspects in diverse vulnerable elderly populations. However, some efforts must be done to strengthen result consistencies from RCTs that study frail older adults. A frail elderly population must be defined based on current knowledge in this field before further conclusions can be drawn. Furthermore, the exercise program must be designed in accordance with current guidelines regarding exercise frequency, duration, and intensity, and it must take into account population heterogeneity (individualization) and other training principles, such as progression and specificity. Experimental researches that focus on long-term exercise adherence in frail older adults are also needed. In sum, exercise training seems to be a safe and effective tool to promote and maintain optimal health levels in a large variety of vulnerable older adults. However, lack of RCTs that utilize a well-defined frail older population based on current knowledge in this field does not permit us to establish that exercise influences health in the elderly. To confirm exercise benefits on health in frail older adults further researches are needed.

Acknowledgments The author thanks Myles Schonekas for help with the English version and Anne-Marie Ferrandez for her important comments.

\section{References}

1. Fried LP, Hadley EC, Walston JD et al (2005) From bedside to bench: research agenda for frailty. Sci Aging Knowledge Environ 31:pe24

2. Walston J, Hadley EC, Ferrucci L et al (2006) Research agenda for frailty in older adults: toward a better understanding of physiology and etiology: summary from the American Geriatrics Society/National Institute on Aging Research Conference on Frailty in Older Adults. J Am Geriatr Soc 54(6):991-1001

3. Passarino G, Montesanto A, De Rango F et al (2007) A cluster analysis to define human aging phenotypes. Biogerontology 8 (3):283-290

4. Bergman H, Hogan DB, Karunananthan S (2007) Frailty: a clinically relevant concept? Canadian Joural of Geriatrics 11:124128

5. Mitnitski A, Song X, Skoog I et al (2005) Relative fitness and frailty of elderly men and women in developed countries and their relationship with mortality. J Am Geriatr Soc 53(12):2184-2189

6. Fried LP, Tangen CM, Walston J et al (2001) Frailty in older adults: evidence for a phenotype. J Gerontol A Biol Sci Med Sci 56(3):M146-M156

7. Chin A Paw MJ, Decker JM, Feskens EJ et al (1999) How to select a frail elderly population? A comparison of three working definitions. J Clin Epidemiol 52(11):1015-1021

8. Lally F, Crome P (2007) Understanding frailty. Postgrad Med J 83 (975): 16-20

9. Hogan DB, MacKnight C, Bergman H (2003) Models, definitions, and criteria of frailty. Aging Clin Exp Res 15(3 Suppl):1-29
10. Fried LP, Ferrucci L, Darer J et al (2004) Untangling the concepts of disability, frailty, and comorbidity: implications for improved targeting and care. J Gerontol A Biol Sci Med Sci 59 (3):255-263

11. Bergman H, Ferrucci L, Guralnik J et al (2007) Frailty: an emerging research and clinical paradigm - issues and controversies. J Gerontol A Biol Sci Med Sci 62(7):731-737

12. Levers MJ, Estabrooks CA, Ross Kerr JC (2006) Factors contributing to frailty: literature review. J Adv Nurs 56(3):282291

13. Chin A Paw MJ, van Uffelen JG, Riphagen I et al (2008) The functional effects of physical exercise training in frail older people: a systematic review. Sports Med 38(9):781-793

14. Binder EF, Schechtman KB, Ehsani AA et al (2002) Effects of exercise training on frailty in community-dwelling older adults: results of a randomized, controlled trial. J Am Geriatr Soc 50 (12):1921-1928

15. Brown M, Sinacore DR, Ehsani AA et al (2000) Low-intensity exercise as a modifier of physical frailty in older adults. Arch Phys Med Rehabil 81(7):960-965

16. Chin A Paw MJ, de Jong N, Schouten EG et al (2001) Physical exercise and/or enriched foods for functional improvement in frail, independently living elderly: a randomized controlled trial. Arch Phys Med Rehabil 82(6):811-817

17. Chin A Paw MJ, de Jong N, Schouten EG et al (2002) Physical exercise or micronutrient supplementation for the wellbeing of the frail elderly? A randomised controlled trial. Br J Sports Med 36 (2):126-131

18. de Jong N, Chin A Paw MJ, de Groot LC et al (2000) Dietary supplements and physical exercise affecting bone and body composition in frail elderly persons. Am J Public Health 90 (6):947-954

19. Ehsani AA, Spina RJ, Peterson LR et al (2003) Attenuation of cardiovascular adaptations to exercise in frail octogenarians. J Appl Physiol 95(5):1781-1788

20. Faber MJ, Bosscher RJ, Chin A Paw MJ et al (2006) Effects of exercise programs on falls and mobility in frail and prefrail older adults: a multicenter randomized controlled trial. Arch Phys Med Rehabil 87(7):885-896

21. Gill TM, Baker DI, Gottschalk M et al (2002) A program to prevent functional decline in physically frail, elderly persons who live at home. N Engl J Med 347(14):1068-1074

22. Greenspan AI, Wolf SL, Kelley ME et al (2007) Tai chi and perceived health status in older adults who are transitionally frail: a randomized controlled trial. Phys Ther 87(5):525-535

23. Greiwe JS, Cheng B, Rubin DC et al (2001) Resistance exercise decreases skeletal muscle tumor necrosis factor alpha in frail elderly humans. Faseb J 15(2):475-482

24. Latham NK, Anderson CS, Lee A et al (2003) A randomized, controlled trial of quadriceps resistance exercise and vitamin D in frail older people: the Frailty Interventions Trial in Elderly Subjects (FITNESS). J Am Geriatr Soc 51(3):291-299

25. Sattin RW, Easley KA, Wolf SL et al (2005) Reduction in fear of falling through intense tai chi exercise training in older, transitionally frail adults. J Am Geriatr Soc 53(7):1168-1178

26. Wolf SL, Sattin RW, Kutner M et al (2003) Intense tai chi exercise training and fall occurrences in older, transitionally frail adults: a randomized, controlled trial. J Am Geriatr Soc 51(12):1693-1701

27. Dorner T, Kranz A, Zettl-Wiedner K et al (2007) The effect of structured strength and balance training on cognitive function in frail, cognitive impaired elderly long-term care residents. Aging Clin Exp Res 19(5):400-405

28. Jensen J, Nyberg L, Rosendahl E et al (2004) Effects of a fall prevention program including exercise on mobility and falls in frail older people living in residential care facilities. Aging Clin Exp Res 16(4):283-292 
29. Rydwik E, Lammes E, Frandin K et al (2008) Effects of a physical and nutritional intervention program for frail elderly people over age 75. A randomized controlled pilot treatment trial. Aging Clin Exp Res 20(2):159-170

30. Shimada H, Uchiyama Y, Kakurai S (2003) Specific effects of balance and gait exercises on physical function among the frail elderly. Clin Rehabil 17:472-479

31. Timonen L, Rantanen T, Makinen E et al (2006) Effects of a group-based exercise program on functional abilities in frail older women after hospital discharge. Aging Clin Exp Res 18(1):50-56

32. Timonen L, Rantanen T, Ryynanen OP et al (2002) A randomized controlled trial of rehabilitation after hospitalization in frail older women: effects on strength, balance, and mobility. Scand J Med Sci Sports 12(3):186-192

33. Timonen L, Rantanen T, Timonen TE et al (2002) Effects of a group-based exercise program on the mood state of frail older women after discharge from hospital. Int J Geriatr Psychiatry 17 (12):1106-1111

34. Wolf SL, Barnhart HX, Kutner NG et al (2003) Selected as the best paper in the 1990s: reducing frailty and falls in older persons: an investigation of tai chi and computerized balance training. J Am Geriatr Soc 51(12):1794-1803

35. Seynnes O, Fiatarone Singh MA, Hue O et al (2004) Physiological and functional responses to low-moderate versus high intensity progressive resistance training in frail elders. J Gerontol A Biol Sci Med Sci 59(5):503-509

36. Wolf SL, O'Grady M, Easley KA et al (2006) The influence of intense Tai Chi training on physical performance and hemodynamic outcomes in transitionally frail, older adults. J Gerontol A Biol Sci Med Sci 61(2):184-189

37. Binder EF, Brown M, Sinacore DR et al (2004) Effects of extended outpatient rehabilitation after hip fracture: a randomized controlled trial. Jama 292(7):837-846
38. Miller MD, Crotty M, Whitehead C et al (2006) Nutritional supplementation and resistance training in nutritionally at risk older adults following lower limb fracture: a randomized controlled trial. Clin Rehabil 20(4):311-323

39. Alexander NB, Galecki AT, Grenier ML et al (2001) Task-specific resistance training to improve the ability of activities of daily living-impaired older adults to rise from a bed and from a chair. J Am Geriatr Soc 49(11):1418-1427

40. Gill TM, Baker DI, Gottschalk M et al (2004) A prehabilitation program for the prevention of functional decline: effect on higher level physical function. Arch Phys Med Rehabil 85(7):1043-1049

41. Rosendahl E, Lindelof N, Littbrand H et al (2006) High intensity functional exercise program and protein-enriched energy supplement for older persons dependent in activities of daily living: a randomised controlled trial. Aust J Physiother 52(2):105-113

42. Nelson ME, Rejeski WJ, Blair SN et al (2007) Physical activity and public health in older adults: recommendation from the American College of Sports Medicine and the American Heart Association. Med Sci Sports Exerc 39(8):1435-1445

43. Rockwood K (2005) Frailty and its definition: a worthy challenge. J Am Geriatr Soc 53(6):1069-1070

44. Ferrucci L, Guralnik JM, Studenski S et al (2004) Designing randomized, controlled trials aimed at preventing or delaying functional decline and disability in frail, older persons: a consensus report. J Am Geriatr Soc 52(4):625-634

45. Kuramoto AM (2006) Therapeutic benefits of Tai Chi exercise: research review. Wmj 105(7):42-46

46. Taylor-Piliae RE, Froelicher ES (2004) Effectiveness of Tai Chi exercise in improving aerobic capacity: a meta-analysis. J Cardiovasc Nurs 19(1):48-57

47. Taylor-Piliae RE (2008) The effectiveness of Tai Chi exercise in improving aerobic capacity: an updated meta-analysis. Med Sport Sci 52:40-53 